\title{
BMJ Open What are the factors that contribute to the development of sexual dysfunction in breastfeeding women? A systematic scoping review protocol
}

Miguel Fuentealba-Torres, ${ }^{1}$ Denisse Cartagena-Ramos, ${ }^{1}$ Juan Carlos Sierra, ${ }^{2}$ Lúcia Alves Lara, ${ }^{3}$ Sérgio Pires Okano, ${ }^{3}$ Thaís Zamboni Berra, ${ }^{1}$ Luana Seles Alves, ${ }^{1}$ Ana Carolina Andrade Biaggi Leite, ${ }^{1}$ Mellina Yamamura, ${ }^{1}$ Isília Aparecida Silva, ${ }^{4}$ Lucila Castanheira Nascimento, ${ }^{1}$ Ricardo Alexandre Arcêncio ${ }^{1}$

To cite: Fuentealba-Torres M, Cartagena-Ramos D, Sierra JC, et al. What are the factors that contribute to the development of sexual dysfunction in breastfeeding women? A systematic scoping review protocol. BMJ Open 2018;8:e22863. doi:10.1136/ bmjopen-2018-022863

- Prepublication history and additional material for this paper are available online. To view these files, please visit the journal online (http://dx.doi. org/10.1136/bmjopen-2018022863).

Received 12 March 2018 Revised 23 June 2018 Accepted 27 July 2018

Check for updates

(c) Author(s) (or their employer(s)) 2018. Re-use permitted under CC BY-NC. No commercial re-use. See rights and permissions. Published by BMJ.

${ }^{1}$ Department of Maternal-Infant and Public Health Nursing,

University of São Paulo, Ribeirão Preto, Brazil

${ }^{2}$ Centro de Investigación Mente, Cerebro y Comportamiento (CIMCYC), University of Granada, Granada, Spain

${ }^{3}$ Department of Gynecology and Obstetrics, University of São Paulo, Ribeirão Preto, Brazil ${ }^{4}$ Department of MaternalInfant and Psychiatric Nursing, University of São Paulo, São Paulo, Brazil

Correspondence to

Mr. Miguel Fuentealba-Torres; mfuentealba@usp.br

\section{ABSTRACT}

Introduction Female sexual dysfunction is a public health problem. Evidence suggests that the population of nursing women is more vulnerable to the phenomenon due to breast feeding. Thus, this protocol was developed to explore the factors that contribute to the development of sexual dysfunction in breastfeeding women.

Methods and analysis The systematic scoping review will be conducted in six stages, according to the Preferred Reporting Items for Systematic Reviews and Meta-

Analyses Protocols. The databases used will be: PubMed, Excerpta Medica Database, Cumulative Index for Nursing and Allied Health Literature, PsycINF0, Science Direct, Web of Science and Google Scholar. The searches were made until 1 June 2018, and no retrospective time limit was used. For the organisation of the literature retrieved from the databases, the EndNote Basic manager will be used. The Cochrane model will be used for the data extraction. The analysis of the quantitative data will be carried out through descriptive statistics and the qualitative data will be submitted to thematic analysis. The methodological quality of the empirical studies will be evaluated using the Mixed Methods Appraisal Tool.

Ethics and dissemination As it will be a review study, without human involvement, there will be no need for ethical approval. The results will be disseminated in a scientific journal, as well as in various media, such as: conferences, seminars, congresses or symposia.

\section{INTRODUCTION}

Sexual dysfunction is a heterogeneous group of disorders, which affects the capacity for sexual response or sexual pleasure. ${ }^{1}$ These disorders have been related to biological, psychological, cultural, behavioural and interpersonal determinants. ${ }^{2}$

Female sexual dysfunction (FSD) is considered a public health problem affecting more than $40 \%$ of the world population of women. ${ }^{3}$ A systematic review estimated that the prevalence of FSD in populations over 18 years
Strengths and limitations of this study

- This protocol reduces the possibility of duplication, gives transparency to the methods and processes that will be used, reduces possible biases and allows peer review.

- This scoping review will be the first to explore the factors that contribute to the development of sexual dysfunction in breastfeeding women.

- The scarcity of empirical studies, the publication bias and the methodological quality of the grey literature found may be the main limitations of the study.

ranged from $5.5 \%$ to $73.2 \%{ }^{4}$, and a meta-analysis estimated that the prevalence of sexual dysfunction among middle-aged women is $40.9 \% .^{5}$

The literature showed that women who breast feed may have a higher risk of sexual dysfunction. $^{4-8} \quad$ Hyperprolactinaemia, hypo-oestrogenism and the demand for infant care may increase FSD in this period..$^{7-12}$ Despite this evidence, few studies have been conducted to identify factors that contribute to the development of FSD among breastfeeding women. ${ }^{13} 14$

Previous studies have investigated sexual dysfunction in the puerperium. ${ }^{9} 13 \quad 15 \quad 16$ However, most of these studies combine populations of women who are breast feeding with populations of women who do not breast feed. This analysis has not allowed to identify specific risk factors for FSD in women who breast feed.

Due to the scarcity of epidemiological studies of FSD in these women, the actual prevalences and risk factors for FSD in this population are still unknown. This panorama has made it impossible, at present, to conduct classic systematic reviews. In spite of this 
Table 1 Structure of the research questions of the systematic scoping review protocol

\begin{tabular}{llll}
\hline Questions & Concept & Target population & Results of interest in health \\
\hline $\begin{array}{l}\text { What happens to female sexual performance } \\
\text { in the course of breast feeding? }\end{array}$ & $\begin{array}{l}\text { Female sexual } \\
\text { performance }\end{array}$ & $\begin{array}{l}\text { Breastfeeding } \\
\text { women }\end{array}$ & $\begin{array}{l}\text { Female sexual performance in the } \\
\text { course of breast feeding. }\end{array}$ \\
$\begin{array}{l}\text { What are the biological and psychosocial } \\
\begin{array}{l}\text { aspects that interfere with the sexual function } \\
\text { of women who breast feed? }\end{array}\end{array}$ & $\begin{array}{l}\text { Biological and } \\
\text { psychosocial } \\
\text { aspects }\end{array}$ & $\begin{array}{l}\text { Breastfeeding } \\
\text { women }\end{array}$ & $\begin{array}{l}\text { Biological and psychosocial aspects } \\
\text { that interfere with the sexual function. }\end{array}$ \\
$\begin{array}{lll}\text { What are the sexual dysfunction problems in } \\
\text { breastfeeding women? }\end{array}$ & $\begin{array}{l}\text { Sexual } \\
\text { dysfunction } \\
\text { problems }\end{array}$ & $\begin{array}{l}\text { Breastfeeding } \\
\text { women }\end{array}$ & $\begin{array}{l}\text { The types of sexual dysfunction } \\
\text { problems. }\end{array}$ \\
\hline
\end{tabular}

situation, it is possible to identify factors that contribute to the FSD of women who breast feed between grey literature, book chapters, studies in development and research with a variety of designs. This study consists of a systematic scoping review developed with the aim of exploring the factors that contribute to the development of FSD in the population of women who breast feed and to promote the development of new research.

\section{METHODS AND ANALYSIS}

This work constitutes a protocol for a systematic scoping review, which will be developed according to the steps proposed by Arksey and O'Malley ${ }^{17}$ and subsequent recommendations. ${ }^{18} 19$ The six stages consist of: (1) identifying the research question; (2) identifying relevant studies; (3) study selection; (4) charting the data ; (5) collating, summarising and reporting results and (6) consultation. $^{1718}$

This protocol follows the Preferred Reporting Items for Systematic Reviews and Meta-Analyses Protocols guidelines (online supplementary file 1) for the bibliographic search process. ${ }^{20}$ Currently, this research is in the second phase, which identifies the relevant studies.

\section{Identifying the research question}

The research questions were discussed by the authors and, from a consensus, three research questions were elaborated, articulated for the purpose of the review. The questions were broad in nature and structured from three components: (1) the definition of the concept to be researched, (2) the target population and (3) the results of interest in health ${ }^{18}$ (table 1$)$.

In this step, the concepts and their definitions to be used to support the questions of this review were also determined (table 2).

\section{Identification of relevant studies \\ Search strategy}

The search strategies were articulated with the research questions and were developed by a team of librarians and researchers with experience in bibliographic searches in databases of the health area. The searches were made until 01 June 2018, and no retrospective time limit was used. The controlled vocabulary terms from the MeSH, APA thesaurus, Cumulative Index for Nursing and Allied Health Literature (CINAHL) subject headings and Entree thesaurus were used in combination with keywords and Boolean operators AND and OR. The search strategies

Table 2 Key concepts and definitions for the study questions

\begin{tabular}{ll}
\hline Concept & Definition \\
\hline $\begin{array}{l}\text { Breastfeeding } \\
\text { women }\end{array}$ & $\begin{array}{l}\text { Breast feeding is when the child receives breastmilk directly from the breasts and the following types are } \\
\text { considered: exclusive breast feeding, breast feeding in combination with fluids, complementary breast } \\
\text { feeding (breast feeding in combination with solids or semisolid). }{ }^{26}\end{array}$ \\
$\begin{array}{l}\text { Sexuality } \\
\text { This included a broad perspective with some conceptual elements of the WHO definition. }{ }^{27} \text { In which, } \\
\text { biological and psychosocial aspects are considered. }\end{array}$ \\
$\begin{array}{ll}\text { Biological } \\
\text { aspects }\end{array}$ & $\begin{array}{l}\text { Consider the interaction of prolactin and oxytocin with female sex hormones including oestrogen and } \\
\text { progesterone. }\end{array}$ \\
$\begin{array}{l}\text { Psychosocial } \\
\text { aspects of } \\
\text { sexuality }\end{array}$ & $\begin{array}{l}\text { It considers the intrapsychic and social aspects of the sexual response of women who breast feed, } \\
\text { including depression, body image, fatigue, sleep deprivation and parental tasks. }\end{array}$ \\
$\begin{array}{l}\text { Female sexual } \\
\text { performance }\end{array}$ & $\begin{array}{l}\text { It is a component of sexual behaviour that is a consequence of the relationship between hormonal actions } \\
\text { in the brain, sexual arousal and expectations of sexual competence that results in the performance of the } \\
\text { individual. }{ }^{28}\end{array}$ \\
$\begin{array}{l}\text { Sexual } \\
\text { dysfunction }\end{array}$ & $\begin{array}{l}\text { Involves a group of disorders that are typically characterised by clinically significant disorders in a person's } \\
\text { ability to respond sexually or experience sexual pleasure. }{ }^{1} \text { These disorders affect the dimensions of desire, } \\
\text { excitement, lubrication, orgasm, satisfaction and pain. }{ }^{29}\end{array}$ \\
\hline
\end{tabular}


were adapted according to the specificities of each database. The filters used were: literature with humans; in the Spanish; Portuguese or English languages; without any time limit or restrictions according to the type of study (online supplementary file 2).

The following multidisciplinary and health sciences electronic databases were used: PubMed, Excerpta Medica Database, CINAHL, PsycINFO, Science Direct and Google Scholar in the search for grey literature. In addition, studies will be identified through the references of the studies retrieved in the databases and selected for the review.

\section{Study selection}

The selection process will be presented through the flow chart Preferred Reporting Items for Systematic Reviews and Meta-Analyses, according to the steps: (1) identification; (2) screening; (3) eligibility and (4) inclusion ${ }^{21}$ (online supplementary file 3 ).

In the identification phase of the literature retrieved, all studies will be organised with the help of the EndNote Basic programme, which manages the organisation, storage, identification and removal of duplicate studies. Then, the references will be exported to a database constructed using Microsoft Excel V.2016.

In the screening stage, the preselection of potentially eligible literature will be performed. In order to reduce potential bias in this step, independent review of the titles and abstracts of the preselected studies will be performed independently by two reviewers (DC-R and MF-T). Using the SPSS V.23.0 software, the interobserver agreement index between the DC-R and MF-T reviewers, using the kappa criterion $>80 \% \quad(\mathrm{p}<0.05)^{22}$ will be calculated. In case of disagreement in the selection of the studies, a third reviewer (RA) will be consulted.

The eligibility stage will be developed by a team of two reviewers (MF-T and DC-R). To avoid possible bias, the reviewers will independently and comprehensively read the literature preselected in the screening stage. In case of disagreement, a third reviewer (RA) will be consulted.

To evaluate the inclusion or exclusion of eligible studies, reviewers will use the following criteria:

\section{Inclusion criteria}

1. Literature in English, Spanish or Portuguese, focused on the research questions detailed in table 1.

2. Literature focusing on sexual dysfunction in nursing population, including: qualitative or quantitative empirical studies; studies of literature review, regardless of the method used and brief communications of ongoing studies.

3. Grey literature focusing on sexual dysfunction in the nursing population, including: annals of congresses, conferences of organisations or societies; academic dissertations, theses, books, book chapters and government documents.
4. Cohort studies focusing on the sexual function of nutritious women, who have followed up from pregnancy to post partum, will also be included.

\section{Exclusion criteria}

Studies focusing on the sexuality of pregnant women and brief communications with inconsistent results will be excluded.

\section{Patient and public involvement}

There was no participation of patients in this study.

\section{Charting the data (extraction of the data)}

In this step, the data will be extracted from the literature included, evaluating the quality of the individual studies selected.

The following data will be extracted based on the Cochrane Consumer and Communication Review Group Data Extraction Template $(2015)^{23}$ : database, year, volume, pages, funding agency, country, language, title, type of study, method, inclusion and exclusion criteria, sample, characteristics of the population, place and period of the study. In addition, information will be extracted regarding the sexual behaviours of women who breast feed, the types of sexual dysfunction and the factors that contribute to FSD (online supplementary file 4).

\section{Mapping of the literature included}

In order to visualise the geographical location of the literature, the articles will be geocoded, through the correspondence address of the first author listed in the article. Through the Google Earth V.7.15 software, the geographical coordinates of these addresses will be obtained and later geocoded using the TerraView V.4.2.2 software. The final file of geocoded addresses will follow the Universal Transverse Mercator projection and the Geocentric Reference System for the Americas 2000 datum. Finally, the articles included in the scoping review will be presented on the map of the world.

\section{Risk of bias (quality) assessment of individual studies}

To reduce the potential bias, the data will be extracted by a team of six reviewers using the standardised extraction form adapted from the Cochrane Consumer and Communication Review Group Data Extraction Template (2016) ${ }^{23}$ The quality of the empirical studies included in the review will be evaluated using the Mixed Methods Appraisal Tool. ${ }^{24}$ This instrument was developed to evaluate the methodological quality of empirical studies included in systematic reviews, conducted through qualitative, quantitative and mixed methods. Subsequently, the evidence of the methodological quality of the scope of the literature will be presented, including the evidence of studies that present low scores in the quality assessment, which will not be excluded from the sample analysed.

\section{Collating, summarising and reporting the data}

The selected studies will be characterised, and the extracted data will be summarised to discuss the evidence 
that will support possible answers to the research questions and recommendations for future studies.

The characterisation of the studies will cover: authors; year of publication; countries; aim of the study; population and sample size; place of study; method; results and evaluation of the methodological quality of empirical studies (online supplementary file 5). For the descriptive analysis of the characteristics of the included literature, the SPSS program is going to be applied calculating absolute frequencies and percentages.

The summary of the quantitative evidence is going to be carried out in a narrative form by identification categories.

The summary of the qualitative evidence will be presented through the themes, constructed from the thematic deductive analysis. The thematic analysis process will be performed through the following steps: (1) familiarisation with the data, (2) generation of initial codes, (3) search for themes, (4) review of themes, (5) definition of the themes and (6) articulation of the themes with the literature of the area and the production of the final analysis. ${ }^{25}$

\section{Stakeholder consultation}

This is the final step of the review and will consist of a panel of experts on the clinical implications of the evidence summarised in the study. ${ }^{18}$

The panel will be made up of three specialists: a researcher with experience in the area of breast feeding; a researcher with psychology and experience in the subject of sexuality and a medical sexologist with experience in research into sexual dysfunction.

In this procedure, a synthesis with the preliminary results of the literature will be sent to the panel of specialists, later, two web-based conferences, approximately 1 hour long, will be scheduled to develop a group discussion about their views on the evidence found in the literature. In the first conference, the practical recommendations of the evidence, dissemination strategies and guidelines for future studies will be discussed. In the second conference, the final report of

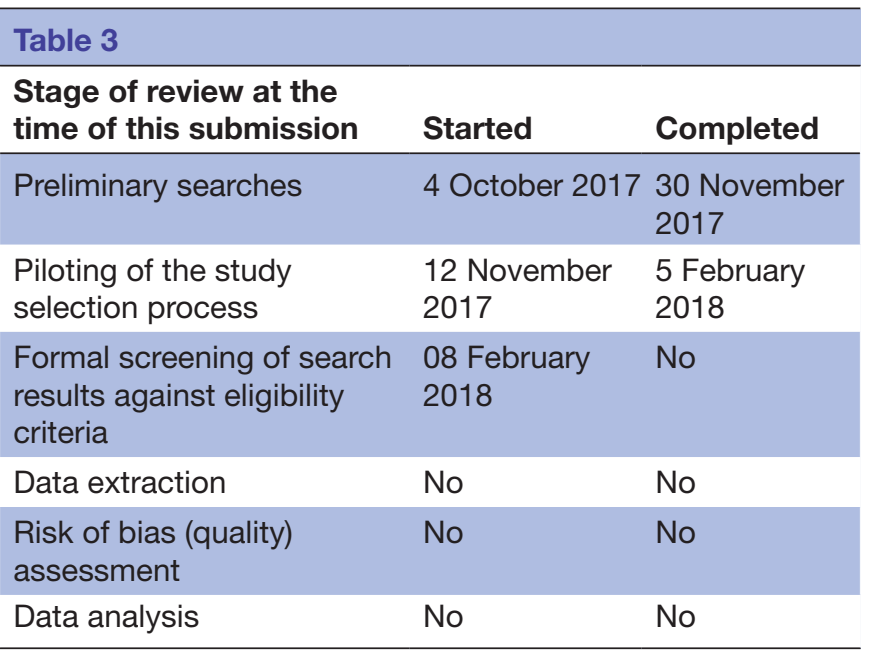

the research will be presented which will conclude with the practical recommendations, dissemination strategies and outlines for future studies contributed by the panel of experts at the first meeting.

\section{ETHICS AND DISSEMINATION}

The results will be disseminated in a scientific journal, as well as in various media, such as: conferences, seminars, congresses or symposia.

The results of the review will be disseminated in an open access journal to ensure access for undergraduate and graduate students, researchers, academics and research groups and will be disseminated at conferences aimed at students, researchers, health teams and nursing women's communities.

The stages of the review will be presented in the following table 3 .

Acknowledgements The authors acknowledge the funding received from the Coordination for the Improvement of Higher Education Personnel (CAPES) and National Council for Scientific and Technological Development (CNPq), PEC-PGProgram, to the first and second authors, as doctoral scholarships.

Contributors As this is a review of the scope of the literature. This review is being conducted by a team of 12 researchers. MF-T, DC-R: proposed the development of the study and coordinated the construction of this protocol. MF-T, DC-R, JCS, LAL, IAS, LCN, RAA: development of the theoretical aspects of method. MF-T, DC-R, JCS, LAL, SPO, TZB, LSA, ACABL, MY, IAS, LCN, RAA: discussed, consensus of the three research questions and articulated for the purpose of the review. MF-T, DC-R, SPO, TZB, LSA, ACABL, MY: performed the systematic search of the seven electronic databases. All authors read and approved the final manuscript.

Funding This research was funded by the Coordination for the Improvement of Higher Education Personnel and National Council for Scientific and Technological Development (CAPES/CNPq), processes numbers 9243143 and 9191134

Competing interests None declared.

Patient consent Not required.

Ethics approval As it will be a review study, without human involvement, there will be no need for ethical approval.

Provenance and peer review Not commissioned; externally peer reviewed.

Open access This is an open access article distributed in accordance with the Creative Commons Attribution Non Commercial (CC BY-NC 4.0) license, which permits others to distribute, remix, adapt, build upon this work non-commercially, and license their derivative works on different terms, provided the original work is properly cited, appropriate credit is given, any changes made indicated, and the use is non-commercial. See: http://creativecommons.org/licenses/by-nc/4.0/.

\section{REFERENCES}

1. American of Psychiatric Association. Diagnostic and statistical manual of mental disorders. 5th edn, 2013.

2. McCabe MP, Sharlip ID, Lewis R, et al. Risk factors for sexual dysfunction among women and men: a consensus statement from the fourth international consultation on sexual medicine 2015. J Sex Med 2016;13:153-67.

3. McCabe MP, Sharlip ID, Lewis R, et al. Incidence and prevalence of sexual dysfunction in women and men: a consensus statement from the fourth international consultation on sexual medicine 2015. J Sex Med 2016;13:144-52.

4. Abdool Z, Thakar R, Sultan AH. Postpartum female sexual function. Eur J Obstet Gynecol Reprod Biol 2009;145:133-7.

5. Convery KM, Spatz DL. Sexuality \& breastfeeding: what do you know? MCN Am J Matern Child Nurs 2009;34:218-23.

6. Leeman LM, Rogers RG. Sex after childbirth: postpartum sexual function. Obstet Gynecol 2012;119:647-55. 
7. Avery MD, Duckett L, Frantzich CR. The experience of sexuality during breastfeeding among primiparous women. J Midwifery Womens Health 2000;45:227-37.

8. Khajehei M, Doherty M, Tilley PJ, et al. Prevalence and risk factors of sexual dysfunction in postpartum Australian women. J Sex Med 2015;12:1415-26.

9. Acele EÖ, Karaçam Z. Sexual problems in women during the first postpartum year and related conditions. J Clin Nurs 2012;21:929-37.

10. Barrett G, Pendry E, Peacock J, et al. Women's sexual health after childbirth. BJOG: An Int Journal Obstet Gynaecol 2000;107:186-95.

11. Rowland M, Foxcroft L, Hopman WM, et al. Breastfeeding and sexuality immediately post partum. Can Fam Physician 2005;51:1366-7.

12. Shirvani MA, Bagheri-Nesami M, Bavand M. P507 Women's sexual dysfunction during the first year after child birth. Int J Gynecol Obstet 2009;107:S557.

13. Anbaran ZK, Baghdari N, Pourshirazi M, et al. Postpartum sexual function in women and infant feeding methods. J Pak Med Assoc 2015;65:248-52.

14. Malakoti J, Zamanzadeh V, Maleki A, et al. Sexual function in breastfeeding women in family health centers of tabriz, iran, 2012. $J$ Caring Sci 2013;2:141-6.

15. Millheiser $\mathrm{L}$. Female sexual function during pregnancy and postpartum. J Sex Med 2012;9:635-6.

16. Woolhouse H, McDonald E, Brown S. Women's experiences of sex and intimacy after childbirth: making the adjustment to motherhood. J Psychosom Obstet Gynaecol 2012;33:185-90.

17. Arksey H, O'Malley L. Scoping studies: towards a methodological framework. Int J Soc Res Methodol 2005;8:19-32.

18. Levac D, Colquhoun H, O'Brien KK. Scoping studies: advancing the methodology. Implement Sci 2010;5:69.
19. Colquhoun HL, Levac D, O'Brien KK, et al. Scoping reviews: time for clarity in definition, methods, and reporting. J Clin Epidemiol 2014;67:1291-4.

20. Shamseer L, Moher D, Clarke M, et al. Preferred reporting items for systematic review and meta-analysis protocols (PRISMA-P) 2015: elaboration and explanation. BMJ 2015;350:g7647.

21. World Health Organization. Indicators for assessing breastfeeding practices. Geneva, Switzerland: World Health Organization, 1991.

22. World Health Organization. Defining Sexual Health: Report of technical consultation on sexual health 28-31 January 2002, Geneva. Sex Heal Doc Ser 2006:30.

23. Pfaus JG. REVIEWS: Pathways of Sexual Desire. J Sex Med 2009;6:1506-33.

24. Wiegel M, Meston C, Rosen R, et al. The Female Sexual Function Index (FSFI): cross-validation and development of clinical cutoff scores. J Sex Marital Ther 2005;31:1-20.

25. Moher D, Liberati A, Tetzlaff $J$, et al. Preferred reporting items for systematic reviews and meta-analyses: the PRISMA statement. PLoS Med 2009;6:e1000097.

26. Mathes T, Klaßen P, Pieper D. Frequency of data extraction errors and methods to increase data extraction quality: a methodological review. BMC Med Res Methodol 2017;17:152.

27. Cochrane Consumer and Communication Review Group. Data extraction template. $2015 \mathrm{http}: / / c c c r g . c o c h r a n e . o r g / a u t h o r-$ resources.

28. Pace R, Pluye P, Bartlett G, et al. Testing the reliability and efficiency of the pilot Mixed Methods Appraisal Tool (MMAT) for systematic mixed studies review. Int J Nurs Stud 2012;49:47-53.

29. Clarke V, Braun V. Teaching thematic analysis: over- coming challenges and developing strategies for effective learning. Psychologist 2013;26:120-3. 